\title{
Clustering of Physical Activities for Quantified Self and mHealth Applications
}

\author{
Chelsea Dobbins \\ Department of Computer Science \\ Liverpool John Moores University \\ Liverpool, UK \\ C.M.Dobbins@1jmu.ac.uk
}

\author{
Reza Rawassizadeh \\ Department of Computer Science and Engineering \\ University of California, Riverside \\ Riverside, CA, USA \\ rrawassizadeh@acm.org
}

\begin{abstract}
The explosion of smaller and more powerful wearable sensing devices has allowed us to continually record and quantify our lives. Undertaking such activities is becoming very popular and has grown into a community called the Quantified Self (QS). Utilizing this outlet has the potential to benefit many aspects of our lives and is gaining momentum within the health sector. However, whilst we can easily collect data, interpreting this information is more challenging. Without extensive data analysis, this information is essentially meaningless in its raw form. This paper posits an approach to quantify and cluster levels of physical activity from accelerometer and heartrate data, which has been obtained from four wearable devices ( 3 accelerometers and 1 hear rate monitor). The approach details our method for pre-processing the data, extracting and selecting the features and a comparison between hierarchical clustering analysis (HCA) and $k$-means. The results illustrate that, whilst both methods are capable of successfully separating the data, the $k$-means approach outperformed the HCA method at clustering the data.
\end{abstract}

Keywords- Quantified Self, mHealth, Physical Activity, Clustering, Heart Rate

\section{INTRODUCTION}

Over the last few decades, technology has advanced at a rapid rate. We are now able to instantly communicate across the globe, track ourselves 24/7 and share our entire lives with the world. Driving such developments has been the Internet, wireless communication, sensor technologies and mobile/wearable devices, which has enabled the formation of a global network, comprised of millions of interconnected devices and objects. Such technology is now ubiquitous and has allowed us to monitor and quantify our lives in ways that were previously unachievable.

One area that has capitalized on this trend has been the Quantified Self $(\mathrm{QS})^{1}$. This movement is comprised of a diverse group of people, including computer scientists, data analysts, health enthusiasts and patients, who subscribe to the concept of "self-knowledge through numbers" [1]. It is this idea that is the driving force behind this movement and has led to people persistently tracking many aspects about themselves. Utilizing a

\footnotetext{
${ }^{1} \mathrm{http}: / /$ quantifiedself.com/
}

range of mobile/wearable devices, we are able to more accurately monitor and quantify our lives, including dietary, behavior and physical activity patterns, through our data. As a result, we can then use this collected data to reflect on our lives and incite behavioral change. May researchers and companies have recognized the power of self-monitoring, in particular for promoting health changes, and have begun incorporating automated sensing applications within self-monitoring technologies [1]. For instance, Apple's ${ }^{\text {TM }}$ iOS 8 saw the release of their Health application ${ }^{2}$. This integrated dashboard retrieves data from the iPhone's ${ }^{\mathrm{TM}}$ on-board accelerometers to display your health and fitness data, including step count, distance and flights of stairs climbed to illustrate how active you've been over the last day, week, month or year. Other devices, such as the Samsung Gear Fit ${ }^{3}$, Nike+ Fuelband ${ }^{4}$ and Fitbit ${ }^{5}$ all track activity through the use of on-board accelerometers. Such devices are also synced with the user's mobile phone so that the collected data can be reflected upon through a dashboard on the adjacent application. However, these devices are still very crude and many are only capable of basic functions, such as counting steps. However, it is crucial and more beneficial for such devices to communicate to the user the types of activities that they have undertaken. In this way, we are more aware of how often certain types of physical activity have been undertaken for and can quantify our intake of physical activity better.

Tracking physical behavior is beneficial to many aspects of our lives and in particular, is gaining momentum within the health community. Being physical inactive is a global issue that is increasing and has been identified as one of the leading risk factors for death around the world [2]. However, manual methods of self-reporting physical activity, such as diaries, are inherently inaccurate and suffer from bias and the fallibility of human memory [3]. In particular, physical activity tends to be overestimated as people overestimate activity duration or

\footnotetext{
${ }^{2}$ https://www.apple.com/uk/ios/whats-new/health/

${ }^{3} \mathrm{http}: / /$ www.samsung.com/uk/consumer/mobile-devices/wearables/gear/SMR3500ZKABTU

${ }^{4}$ https://secure-nikeplus.nike.com/plus/products/fuelband/

${ }^{5}$ https://www.fitbit.com/uk
} 
misjudge inactive or light activity as being moderate [4]. However, recent advances in technology can alleviate this problem, to a certain extent. Over the last decade, the phrase mHealth (mobile health) has emerged as a new paradigm within the healthcare sector. mHealth utilizes mobile/wearable computing, the internet, wireless communication and medical sensing to deliver and support healthcare ubiquitously [5].

Given the nomadic and connectivity nature of smartphones and wearables, these outlets provide an ideal platform to build upon so that we can support the delivery of mHealth in a number of ways [6]. For instance, a QS system that monitors and gathers data, over a sustained period of time could significantly improve the prevention, diagnosis and treatment of several noncommunicable diseases, including obesity and depression [7], [8]. As the system gathers more information about the user (e.g. physiological data), it can "learn" about their lifestyle. These patterns of behavior can then be analyzed to recommend healthy lifestyle changes and the user can also use this information to reflect on their levels of activity to improve the quality of life [9].

However, whilst wearable and mobile devices can easily collect data, interpreting this information is more challenging. Without extensive data analysis, this information is essentially meaningless in its raw form. This paper posits an approach to quantify levels of physical activity from 1) accelerometer and 2) heartrate data, which has been obtained from four wearable devices; 3 accelerometers located around the chest and on the dominant wrist and ankle and a heart rate monitor around the chest. In order to achieve this, the raw data has first been transformed into a set of features. This enables a large set of data to be condensed into a more manageable size, which can then be used within the clustering algorithm. The approach is being used to test the system's ability in separating data into higher and lower occurrences of activity. This information can then be used by patients and care givers to gain a greater insight into the activities of the user by enabling their actions to be quantified in a more accurate manner. In this way, our approach can determine the frequency of high-energy (e.g. running) and low-energy (e.g. sitting) activities, as opposed to simply counting steps/distance.

The reminder of this paper is constructed as follows. Section 2 describes previous related works, while section 3 illustrates the materials and methods that have been used to pre-process the data and extract the features, as well as the clustering process. Section 4 presents the results of the data evaluation and a discussion of the findings. The paper is then concluded in section 5 and future directions of the research are presented.

\section{RELATED WORK}

The widespread availability and lower cost of sensors has enabled the Quantified Self (QS) community to expand [10]. This group is a worldwide collaboration of users (academic, industrial and the general public) who are interested in self-tracking tools. They hold regular international meetings, conferences and expositions to discuss self-tracking technologies and advancements in the field. As a result, there have been many approaches and studies that have been undertaken to explore this area of personal data collection and activity recognition [11][16].

Habib ur Rehman et al. [17] present a taxonomy for personal data mining in resource-constrained environments (e.g. smartphones). Their results illustrate that mining personal data for patterns has a great potential in monitoring the physiologies of users and in the diagnosis of irregularities in a user's daily life. In other works, Dias and Machado da Silva [18] have created a wearable textile prototype, consisting of leggings that are embedded with eight sensors to capture kinematic information. In other works, Lee and Shiroma [19] report the issues and challenges of using accelerometers to measure physical activity. After undertaking a large scale epidemiologic study, which relied on the participants wearing the accelerometers for a week, they reported on the logistical, data reduction and analysis challenges that they faced. Such issues included participants not wearing/returning the devices, the size of the datasets that were produced ( 20 terabytes) and issues relating to the "correct" cut points for classifying sedentary behavior. Uddin et al.'s [20] wearable sensing framework utilizes a 9-axis wristband to continuously monitor a users' daily activities. The data has then been preprocessed and segmented before being passed to the activity recognition algorithm. In other works, Saeedi et al. [21] have developed an automatic on-body sensor platform, consisting of accelerometers and gyroscope sensors, to monitor physical activity.

Similarly, Qiu et al. [22] use accelerometer data, from a SenseCam, and machine learning tools to automatically identify user activity. In their approach, a Support Vector Machine (SVM) is trained to automatically classify accelerometer features into user activities (sitting or standing, driving, walking or lying down) [22]. The accuracy of each activity ranged from $90 \%$ to $98 \%$. This work illustrates how machine learning, and a wearable accelerometer can be used to identify the activities of a wearer to a very high accuracy [22]. Nevertheless, whilst these results are encouraging, this work only considers one type of data, acceleration. However, our approach uses a combination of acceleration and heart rate data. This enables us to better quantify the activities of the user.

\section{MATERIALS AND METHODS}

As sensing devices become smaller and more widely available, this presents us with unique opportunities to collect and analyze data so that QS technologies can move forward into intelligent systems that can learn and react to the user. Utilizing a data processing flow to process accelerometer and heartrate data signals (see Fig. 1), our approach is capable of separating instances of user activities in clusters of higher and lower energy. By successfully separating such occurrences enables a clear pattern of behavior to be established. 


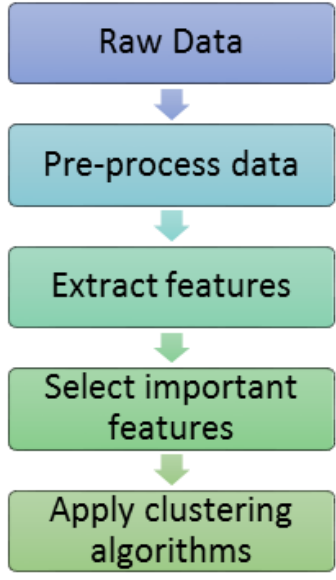

Fig. 1. Our proposed accelerometer and heart rate data processing flow

Our approach focuses on using widely available accelerometer and heartrate sensors, which are extensively used in the area of activity recognition [5], [18], [19]. In this sense, raw data from three wearable tri-axil accelerometers and a heartrate monitor have been obtained from the PAMAP2 Physical Activity Monitoring Data Set [16]. The accelerometers have been placed on the user's dominant wrist and ankle, as well as on the chest. The sampling frequency of the accelerometers was $100 \mathrm{~Hz}$, whilst the heart-rate monitor was $9 \mathrm{~Hz}$.

The data has been recorded from seven subjects. Each volunteer undertook a series of nine activities that are a blend of inactive/low states (e.g. sitting) and highly activity (e.g. running). Each subject adhered to the data collection protocol, which included performing each activity for up to three minutes, with one-minute breaks. Table I lists the level of activity and associated activities that were performed for each category. In total, the dataset contains $13,524,350$ raw records. Once the data has been collected, the signals will be pre-processed before features have been extracted and selected.

\section{A. Data Pre-processing}

The accelerometer data has been normalized using a secondorder forward-backward digital low-pass Butterworth filter, with a cut-off frequency of $3 \mathrm{~Hz}$. A sliding average window of 512 samples, with a $50 \%$ overlap (256 samples), has also been applied to the data so that the records can be reduced, without losing information.

\section{B. Feature Extraction}

Features have then been extracted from the raw data. This has been undertaken in two different modes, time and frequency. These two modes differ as time domain analysis measures the signal over the period of the recording. In contrast, frequency domain analysis depicts how the signal's energy is distributed over a range of frequencies [23]. As such, frequency domain techniques have been extensively used to capture the repetitive nature of a sensor signal. This repetition often correlates to the periodic nature of a specific activity such as
TABLE I. SUMMARY OF THE ACTIVITIES THAT HAVE BEEN PERFORMED BY THE PARTICIPANTS [16]

\begin{tabular}{|c|c|}
\hline $\begin{array}{c}\text { Level of Physical } \\
\text { exertion }\end{array}$ & Activity \\
\hline & Lying \\
\hline Light & Sitting \\
& Standing \\
Ironing \\
\hline Moderate & Descending Stairs \\
& Vacuum Cleaning \\
Normal Walking
\end{tabular}

walking or running [24]. The advantage of frequency-related parameters is that they are less susceptible to signal quality variations [25]. Utilizing the mathematical operators Fast Fourier Transform (FFT) and Power Spectral Density (PSD) the raw signal has been converted between these two modes. From the time domain, the mean, median, standard deviation, root mean square (RMS), variance and correlation have been calculated for the accelerometer signals. From the heart rate monitor, the mean of this signal has also been determined. From the frequency domain, energy, entropy, peak frequency and median frequency have been calculated for the accelerometer signals. This has been confirmed by analyzing the literature [11], [13], [14], [16].

These features comprise the complete original feature set. Each accelerometer that was placed on the ankle, chest and hand has its own feature vector (e.g. ankle_mean, chest_mean, hand_mean, ankle_energy, chest_energy, hand_energy, etc.). In the Time Domain 1,197 feature records have been generated, whilst the Frequency Domain contains 756 records, thus totaling 1,953 records.

\section{Feature Selection}

Whilst a range of features have been generated, some may be redundant. In this instance, dimensionality reduction, utilizing Principal Component Analysis (PCA), has been performed to find a subsection of the most important features. The features have been analyzed in terms of "blocks". For example, all of the median feature vectors (ankle_median, chest_median, hand_median) have utilized PCA. During the next iteration, all of the root mean square vectors have been processed (ankle_rms, chest_rms, hand_rms). This process has been repeated until all of the features have been processed, and the top two components with the best discriminant capabilities have been chosen. Fig. 2 illustrates an example of one PCA graph that has been generated per feature set. Each feature in the bi-plot (see Fig. 2) are represented as an eigenvector and the direction and length of the vector (blue line) indicates how each variable contributes to the principal components in the plot. The feature closest to the horizontal axis, of Fig. 2 shows that in this instance ankle_median is nearest to the principal component, which has 


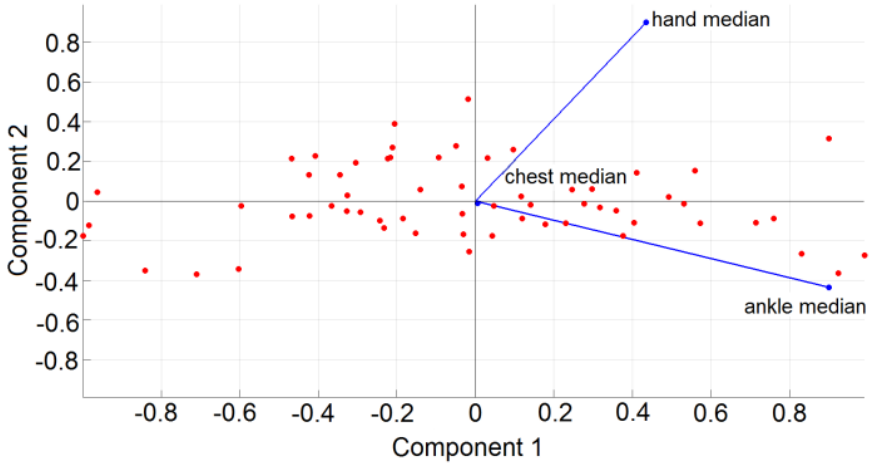

Fig. 2. An example of one principle component analysis graph that has been generated for the median features

the most discriminant capabilities of the considered features. The feature closest to the vertical axis illustrates hand_median as the second component with very good discriminant capabilities. Once the top two features of each set have been selected, it is still unclear if this is the optimal number of features. In this instance, we have used a scree plot to overcome this and to dispute or validate the number of features that have been selected via PCA (see Fig. 3).

This graph plots the generated eigenvalues and arranges them in descending order. The point at which the curve of decreasing eigenvalues decelerates to a flat slope (also known as the "elbow") is the cutoff point and determines the number of features to use. Fig. 3 illustrates the generated scree plot of the data set. As it can be seen, after four features, the "elbow" of the graph appears and the plot trails off to zero. This indicates that out of the PCA features, four of them have the best discriminative capabilities to represent the entire dataset. In summary, PCA has established that ankle_root_mean_square, ankle_mean and hand_mean, hand_root_mean_square, are the best features to use in the study. This new dataset of features is now known as the ClustFeature set and now includes only the features that contain the most information, which are highly associated with each other.

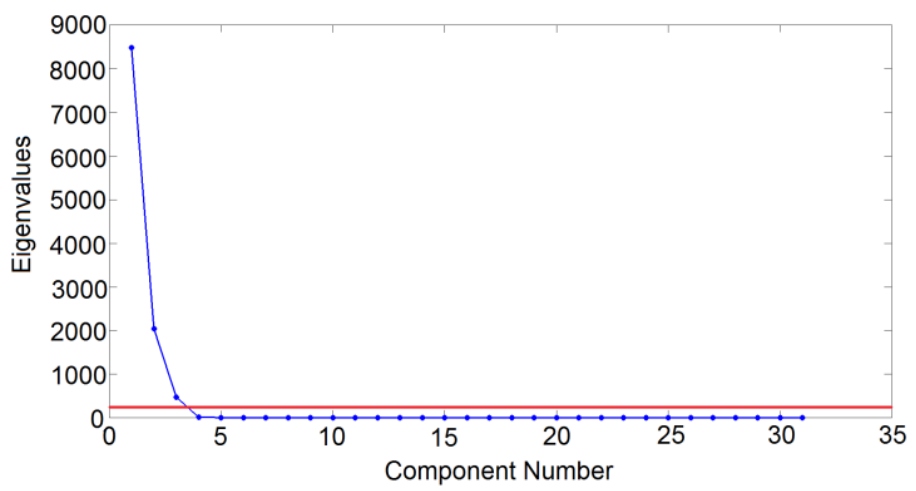

Fig. 3. Scree Plot of the feature set. The graph illsutrates that after four features, the "elbow" of the graph appears
TABLE II. SILHOUETTE AVERAGES FOR $K$ CLUSTERS

\begin{tabular}{|c|c|}
\hline Cluster $(\boldsymbol{k})$ & Silhouette Average (SA) \\
\hline 2 & 0.6492 \\
\hline 3 & 0.6442 \\
\hline 4 & 0.6037 \\
\hline 5 & 0.5312 \\
\hline
\end{tabular}

\section{Clustering}

Clustering methods can be divided into two main groups: hierarchical and partitioning. The hierarchical approach constructs the clusters by recursively partitioning the instances in either a top-down or bottom-up fashion, whereas partitioning relocates instances by moving them from one cluster to another, starting from an initial partitioning. Following an analysis of the literature, the techniques that have been selected for our evaluation include agglomerative (hierarchical) and $k$-means (partitioning) algorithms.

The $k$-means approach requires the user to define the number of clusters $(k)$ beforehand. The centroid is the mean position of the clusters and this is then initialized. Each object is then assigned to its nearest centroid (cluster) and the mean of the new centroids (clusters) are then calculated. This process is repeated until the centroids (clusters) don't change. However, defining the number of clusters $(K)$ can be challenging. In this instance, calculating the silhouette averages can be used to overcome this issue. This value is used as a measurement of the quality of the resulting clusters. The value of $k$ that has the largest SA indicates the most appropriate value to use.

Using this method, the value of $\mathrm{k}$ has been increased, from 2 to 5 , and evaluated using the silhouette function. Table II illustrates the SA's of the various cluster sizes for the dataset. As it can be seen in this table, for this dataset, the most appropriate number of clusters to use is 2 . As a results $k=2$ will be used within the $k$-means results.

\section{RESULTS AND DISCUSSION}

This section presents the results that have been obtained from our approach to cluster the data using Hierarchical Clustering Analysis (HCA) and k-means. Each clustering algorithm is being evaluated on its ability to separate instances into periods of high/low energy so that we can quantify the user's activities. For example, a large cluster of lower energy activities would indicate that they have been particularly inactive over a given period of time.

Fig. 4 illustrates the results of the k-means analysis. In this graph, the center of each cluster has been marked by the "Centroids" symbol. As it can be seen, there is a clear divide in the data. After analyzing the results, the majority of the activities are located within cluster 2 and relate to higher energy activities, such as walking, running and ascending stairs. Cluster 1 refers to lower energy activities, such as sitting, descending stairs and 
vacuum cleaning. Since cluster 2's activities do exert more energy than cluster 1, the algorithm has correctly grouped the information into activities that have higher energy, since walking, running and ascending stairs use additional energy as opposed to descending stairs and vacuum cleaning. This has been confirmed by analyzing the dataset directly.

Fig. 5 and Fig. 6 demonstrates the results of the Hierarchical Clustering Analysis (HCA) algorithm. Fig. 5 is the resulting dendrogram. As it can be seen, this algorithm has separated the data into three main clusters. Fig. 6 illustrates the results of Fig. 5 in a scatter diagram. In comparison to the $k$-means results in Fig. 4, the pattern of the cluster in Fig. 6 is similar, however HCA has partitioned the data differently. The majority of the activities are located within cluster 3 and relates to running, walking, ascending and descending stairs and vacuum cleaning. Cluster 1 contains one instance of walking, ascending and descending stairs and vacuum cleaning, whilst cluster 2 is primarily composed of descending stairs and vacuum cleaning. This has been confirmed by analyzing the dataset directly. Whilst the pattern of the clusters is similar to the k-means approach in Fig. 4 , the data has not been separated correctly as cluster 1 only has one instance of each activity. This approach has not been as effective as $k$-means.

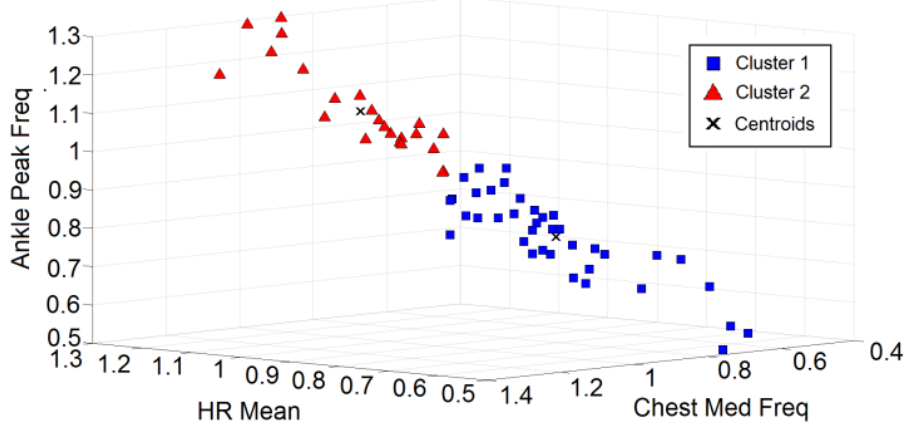

Fig. 4. Clustering results of the data using k-Means Analysis

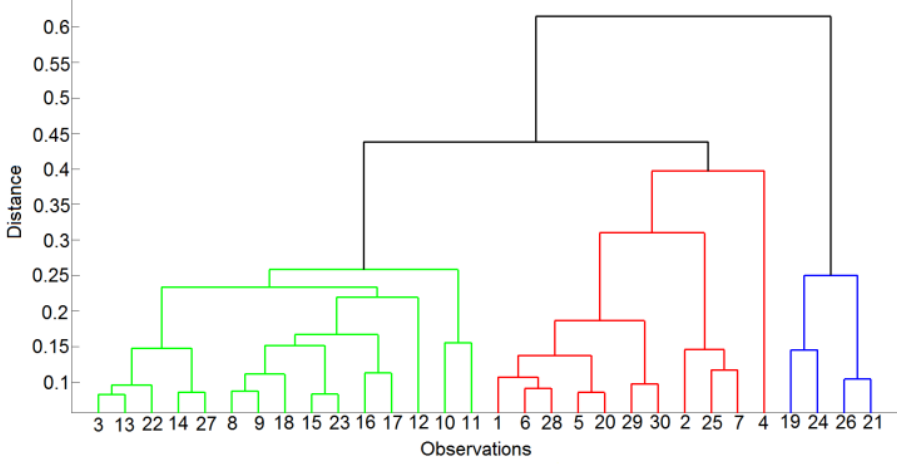

Fig. 5. Clustering results of the data using Hierarchical Clustering Analysis (HCA)

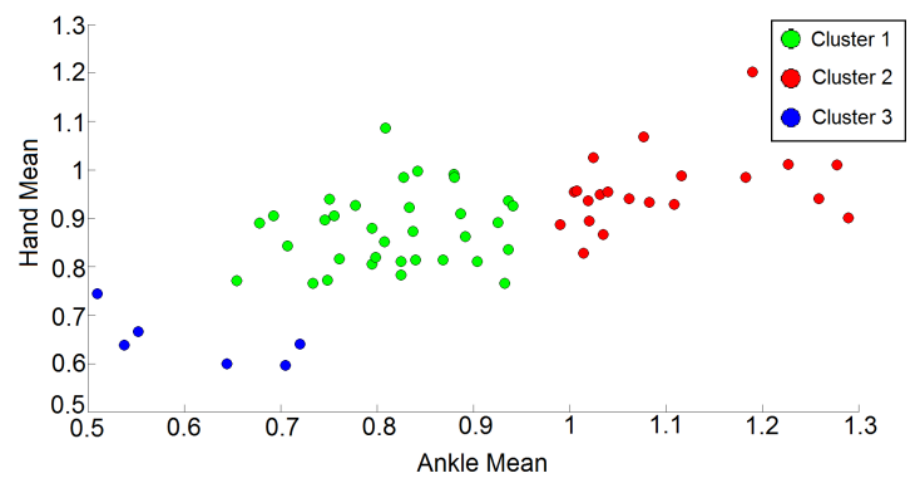

Fig. 6. Scatter plot of the HCA results from Fig. 5

\section{A. Discussion}

The approach posited in this paper is seen as a technique to quantify physical activity data so that patients and care givers can gather a more accurate overview of activity data. Currently, other works, such as [20]-[22] only consider the data from accelerometers. However, this is in contrast to our approach that supports multimodal inputs in the form of acceleration and heart rate data. This enables us to better quantify the activities of the user and to illustrate how such signals can be combined together. The results have provided positive results. These results can be used to determine, for example, significant periods of inactivity. For example, in the case of a patient suffering from obesity, their doctor may advise them to undertake more physical activity and to keep track of their progress. However, as previously discussed, accurately recording such data, using manual methods, such as diaries, can be quite inaccurate. However, using our approach, if the care giver had access to such data they could see that the patient may not be engaging in enough physical activity.

Providing a comparison between the $k$-means and hierarchical results also proved interesting. The hierarchical approach was not as effective in separating the data and provided some anomalies in terms of the clusters that were produced. Hierarchical clustering does not require the user to input the number of cluster that they require. This resulted in the creation of very small clusters that only contained a couple of instances, which was not statistically significant. However, calculating the silhouette values, as in the $k$-means approach, enables the data to be quantified in terms of the similarity between objects to discern the appropriate number of clusters. This measure of quality has enabled the $k$-means approach to produce better results. Nevertheless, it was interesting that the shapes of the clusters between the two approaches were very similar, even if the data has been analyzed differently. This could be attributed to how the clustering algorithms separate. The hierarchical approach constructs the clusters by recursively partitioning the instances in either a top-down or bottom-up fashion, whereas $k$-means relocates instances by moving them from one cluster to another, starting from an initial partitioning. However, in terms of this data, $k$-means performed better at separating the data. 
C. Dobbins and R. Rawassizadeh, "Clustering of Physical Activities for Quantified Self and mHealth Applications," in The 14th IEEE International Conference on Ubiquitous Computing and Communications (IUCC-2015): First International Workshop on Mobile Technology for Healthcare (MT4H 2015), 2015 (Accepted).

\section{SUMMARY AND FUTURE WORK}

This paper has posited our approach to quantify physical activity for use within mHealth applications. In this sense, the system is able to separate instances of high and low energy to determine the user's overall level of activity, during a given period. In achieving this, the methodology that has been used to pre-process raw accelerometer data has been discussed. Features have then been extracted and analyzed, using a variety of methods, including PCA. Overall, the system provides a promising and flexible solution, which can be extended in the future. Its application has the potential to influence our lives by providing a method of learning about ourselves through our data.

However, whilst we can quantify our activity data, future work would consider incorporating other pieces of data, such as photos and location of events so that a visual illustration of our activities can begin to emerge. Additionally, testing the algorithms ability at separating similar activities would also be further line of enquiry. For instance, determining how well we can separate high energy activities into periods of intense physical exertion and those that require some movement. Furthermore, allowing the user to question their data, such as "How many hours do I watch TV?" or "How many hours do I sit at work?" would be interesting.

\section{REFERENCES}

[1] E. K. Choe, N. B. Lee, B. Lee, W. Pratt, and J. A. Kientz, "Understanding Quantified-Selfers' Practices in Collecting and Exploring Personal Data," in Proceedings of the 32nd Annual ACM Conference on Human Factors in Computing Systems (CHI'14), 2014, pp. 1143-1152.

[2] World Health Organization, "Global recommendations on physical activity for health," World Health Organization, 2010.

[3] P. Kelly, A. Doherty, E. Berry, S. Hodges, A. M. Batterham, and C. Foster, "Can We Use Digital Life-Log Images To Investigate Active And Sedentary Travel Behaviour? Results From A Pilot Study," Int. J. Behav. Nutr. Phys. Act., vol. 8, no. 1, p. 44, Jan. 2011.

[4] R. P. Troiano, D. Berrigan, K. W. Dodd, L. C. Mâsse, T. Tilert, and M. McDowell, "Physical Activity in the United States Measured by Accelerometer," Med. Sci. Sports Exerc., vol. 40, no. 1, pp. 181-188, Jan. 2008.

[5] R. Rawassizadeh, B. A. Price, and M. Petre, "Wearables: Has the Age of Smartwatches Finally Arrived?," Commun. ACM, vol. 58, no. 1, pp. 45-47, 2014.

[6] P. Fergus, S. Iram, D. Al-Jumeily, M. Randles, and A. Attwood, "Home-Based Health Monitoring and Measurement for Personalised Healthcare," J. Med. Imaging Heal. Informatics, vol. 2, no. 1, pp. 3543, 2012.

[7] P. Bonato, "Wearable Sensors and Systems. From Enabling Technology to Clinical Applications," IEEE Eng. Med. Biol. Soc. Mag., vol. 29, no. 3, pp. 25-36, Jan. 2010.

[8] A. L. Lopresti, S. D. Hood, and P. D. Drummond, "A review of lifestyle factors that contribute to important pathways associated with major depression: Diet, sleep and exercise," J. Affect. Disord., vol. 148, no. 1, pp. 12-27, May 2013.

[9] R. Rawassizadeh, M. Tomitsch, K. Wac, and A. M. Tjoa, "UbiqLog: a generic mobile phone-based life-log framework," Pers. Ubiquitous Comput., vol. 17, no. 4, pp. 621-637, Apr. 2012.

[10] E. K. Choe, N. B. Lee, B. Lee, W. Pratt, and J. A. Kientz, "Understanding Quantified-Selfers' Practices in Collecting and Exploring Personal Data," in Proceedings of the 32nd Annual ACM
Conference on Human Factors in Computing Systems (CHI '14), 2014, pp. 1143-1152.

[11] P. Srivastava and W.-C. Wong, "Hierarchical Human Activity Recognition Using GMM," in AMBIENT 2012: The Second International Conference on Ambient Computing, Applications, Services and Technologies, 2012, no. c, pp. 32-37.

[12] F. Mokaya, B. Nguyen, C. Kuo, Q. Jacobson, A. Rowe, and P. Zhang, "MARS: A Muscle Activity Recognition System Enabling Selfconfiguring Musculoskeletal Sensor Networks," in The 12th ACM/IEEE Conference on Information Processing in Sensor Networks (IPSN), 2013, pp. 191-202.

[13] S. Abdullah, N. D. Lane, and T. Choudhury, "Towards Population Scale Activity Recognition: A Framework for Handling Data Diversity," in Twenty-Sixth AAAI Conference on Artificial Intelligence, 2012, pp. 851-857.

[14] W. Song, C. Ade, R. Broxterman, T. Barstow, T. Nelson, and S. Warren, "Activity Recognition in Planetary Navigation Field Tests Using Classification Algorithms Applied to Accelerometer Data," in 2012 Annual International Conference of the IEEE Engineering in Medicine and Biology Society (EMBC), 2012, vol. 2012, pp. 15861589.

[15] A. Mannini and A. M. Sabatini, "Machine learning methods for classifying human physical activity from on-body accelerometers.," Sensors, vol. 10, no. 2, pp. 1154-1175, Jan. 2010.

[16] A. Reiss and D. Stricker, "Creating and Benchmarking a New Dataset for Physical Activity Monitoring," in The 5th Workshop on Affect and Behaviour Related Assistance (ABRA), 2012.

[17] M. Habib ur Rehman, C. S. Liew, T. Y. Wah, J. Shuja, and B. Daghighi, "Mining Personal Data Using Smartphones and Wearable Devices: A Survey,” Sensors, vol. 15, no. 2, pp. 4430-4469, Jan. 2015.

[18] R. Dias and J. Machado da Silva, "A Flexible Wearable Sensor Network for Bio-Signals and Human Activity Monitoring," in 2014 11th International Conference on Wearable and Implantable Body Sensor Networks Workshops, 2014, pp. 17-22.

[19] I.-M. Lee and E. J. Shiroma, "Using Accelerometers to Measure Physical Activity in Large-Scale Epidemiological Studies: Issues and Challenges," Br. J. Sports Med., vol. 48, no. 3, pp. 197-201, Feb. 2014.

[20] M. Uddin, A. Salem, I. Nam, and T. Nadeem, "Wearable Sensing Framework for Human Activity Monitoring," in Proceedings of the 2015 workshop on Wearable Systems and Applications, 2015, pp. 2126.

[21] R. Saeedi, J. Purath, K. Venkatasubramanian, and H. Ghasemzadeh, "Toward Seamless Wearable Sensing: Automatic On-Body Sensor Localization for Physical Activity Monitoring," in 36th Annual International Conference of the IEEE Engineering in Medicine and Biology Society (EMBC), 2014, pp. 5385-5388.

[22] Z. Qiu, A. R. Doherty, C. Gurrin, and A. F. Smeaton, "Mining User Activity as a Context Source for Search and Retrieval," in 2011 International Conference on Semantic Technology and Information Retrieval (STAIR), 2011, pp. 162-166.

[23] A. Haensel, P. J. Mills, R. A. Nelesen, M. G. Ziegler, and J. E. Dimsdale, "The relationship between heart rate variability and inflammatory markers in cardiovascular diseases," Psychoneuroendocrinology, vol. 33, no. 10, pp. 1305-1312, Nov. 2008.

[24] D. Figo, P. C. Diniz, D. R. Ferreira, and J. M. P. Cardoso, "Preprocessing Techniques for Context Recognition from Accelerometer Data," Pers. Ubiquitous Comput., vol. 14, no. 7, pp. 645-662, Mar. 2010.

[25] W. L. Maner, R. E. Garfield, H. Maul, G. Olson, and G. Saade, "Predicting term and preterm delivery with transabdominal uterine electromyography.," Obstet. Gynecol., vol. 101, no. 6, pp. 1254-1260, Jun. 2003. 